\title{
Stroke Thrombectomy in Patients with COVID-19: Initial Experience in 13 Cases
}

(D) Rop, (D) A. Hasiu, (D). Bolognini, (DD. Mihoc, (D). Quenardelle, (D). Gheoca, (D)E. Schluck, (D). Courtois, (D). Delaitre, (D) M. Musacchio, (D). Pottecher, (D)T.-N. Chamaraux-Tran, (D). Sellal, (D) V. Wolff, DP.A. Lebedinsky, and (D) R. Beaujeux

\section{ABSTRACT}

SUMMARY: We performed a retrospective review in both comprehensive stroke units of a region affected early by the coronavirus disease 2019 (COVID-19) pandemic, between March 1 and April 26, 2020, including patients with COVID-19 who underwent mechanical thrombectomy for ischemic stroke. We identified 13 cases, representing $38.2 \%$ of 34 thrombectomies performed during this period. We observed increased mortality and a high incidence of thrombotic complications during hospitalization. Given the high rate of infected patients, systematic use of full personal protection measures seems justified.

ABBREVIATIONS: COVID-19 = coronavirus disease 2019; SARS-CoV-2 = Severe Acute Respiratory Syndrome coronavirus 2

$\mathrm{T}$ he coronavirus disease (COVID-19) pandemic is an ongoing pandemic caused by Severe Acute Respiratory Syndrome coronavirus 2 (SARS-CoV-2). The outbreak was identified in China in December 2019 and then spread to Europe in January 2020. The first 3 cases in Europe were reported in France on January $24{ }^{1}$ Epidemic response measures were triggered in France on March 1 and gradually developed until March 17, when a nationwide lockdown was enforced, with strict home confinement of the entire population.

Alsace was the first French region affected by the outbreak. It is a region in the East of France with a population of approximately 1.9 million people. The peak of the epidemic was registered on April 2, with 2142 hospitalized patients diagnosed with COVID-19 (of whom 433 were in intensive care units). On April 26 , there were 1153 reported in-hospital deaths. ${ }^{2}$

We aimed to describe baseline characteristics, procedural metrics, and outcomes of stroke thrombectomies in patients diagnosed with COVID-19, performed at the 2 comprehensive stroke centers (Strasbourg University Hospital and Colmar Hospital) in the Alsace region during the first 2 months of the outbreak.

Received May 17, 2020; accepted after revision June 26.

From the Interventional Neuroradiology Department (R.P., A.H., D.M., R.B.), Stroke Unit (V.Q., R.G., V.W.), and Department of Anesthesia (J.P., T.-N.C.-T.), Strasbourg University Hospitals, Strasbourg, France; Institut de Chirurgie Minime Invasive Guidée par l'Image (R.P., R.B.), Strasbourg, France; Diagnostic and Interventional Neuroradiology Department (F.B., M.D., M.M., P.A.L.) and Neurology Department (F.S.), Hôpitaux Civils de Colmar, Colmar, France; and Neurology Department (E.S., S.C.), Emile Muller Hospital, Mulhouse, France.

Please address correspondence to Raoul Pop, MD, Interventional Neuroradiology Department, Strasbourg University Hospitals, Strasbourg, France, 1 Ave Molière, 67098 Strasbourg, France; e-mail: pop.raoul@gmail.com; @RaoulPop25

- Indicates open access to non-subscribers at www.ajnr.org

http://dx.doi.org/10.3174/ajnr.A6750

\section{MATERIALS AND METHODS}

We performed a retrospective review in both comprehensive stroke units in the Alsace region, between March 1 and April 26, 2020. Patients were included in the study if they underwent mechanical thrombectomy for acute ischemic stroke and were diagnosed with SARS-Cov-2 infection either before or after the endovascular procedure (during the same hospitalization). Endovascular procedures for cerebral venous sinus thrombosis were not included in this study. Patient files were reviewed to extract demographics, comorbidities, procedural metrics, and complications, as well as clinical follow-up information. In addition, we collected protocols of personal protection measures in the angiography suites and the number of documented contamination cases among medical personnel.

\section{Brief Description of the Stroke Pathway in the Alsace Region}

There are 3 hospitals (Strasbourg University Hospital, Colmar Hospital, and Moulhouse Hospital) with stroke units in the region, of which 2 have endovascular treatment facilities (Strasbourg University Hospital and Colmar Hospital). Two additional hospitals (Saverne Hospital and Haguenau Hospital) without stroke units are equipped with telemedicine facilities in the emergency department and are connected to one of the comprehensive stroke centers. Patients are accepted as with stroke alerts following a telephone consultation between the paramedic/ physician in the field and the on-call neurologist if there are symptoms suggestive of stroke dating 6 hours from last seen well (up to 24 hours in selected cases). Patients with stroke alerts bypass the emergency department and are seen directly by the on-call neurologist in the radiology department before imaging. 


\begin{tabular}{lc}
\hline & $\begin{array}{c}\text { Thrombectomies in Patients } \\
\text { with COVID-19 ( } \boldsymbol{n}=13)\end{array}$ \\
\hline Age (median) (IQR) (yr) & $78(71-79)$ \\
Male sex (No.) (\%) & $5(38.4 \%)$ \\
Risk factors (No.) (\%) & \\
Hypertension & $8(61.5 \%)$ \\
Dyslipidemia & $3(23.0 \%)$ \\
Diabetes & $2(15.3 \%)$ \\
Smoking & $3(23.0 \%)$ \\
Baseline mRS score >2 & $0(0 \%)$ \\
Baseline NIHSS score (median) (IQR) & $13(7-15)$ \\
Symptom onset to femoral puncture (median) (IQR) (min) & $244(232-290)$ \\
IV thrombolysis (No.) (\%) & $4(30.7 \%)$ \\
Initial imaging type (No.) (\%) & \\
MR imaging & $11(84.6 \%)$ \\
CT & $2(15.3 \%)$ \\
Initial APECTS & \\
ASPECTS $<5$ (n/N) (\%) & \\
ASPECTS score (median) (IQR) & $0 / 12(0 \%)$ \\
Occlusion site at angiography (No) (\%) & $8(5.7-9)$ \\
M1 segment & \\
M2 segment & $8(61.5 \%)$ \\
Tandem occlusion & $3(23.0 \%)$ \\
Basilar trunk & $1(7.6 \%)$ \\
\hline
\end{tabular}

Note:-IQR indicates interquartile range; $n / N, n$ - number of cases with ASPECTS $<5 / N$ - number of cases with anterior circulation strokes.

${ }^{a}$ For anterior circulation strokes.

Table 2: Thrombectomy procedure metrics: clinical and radiologic outcomes

\begin{tabular}{|c|c|}
\hline & $\begin{array}{l}\text { Thrombectomies in Patients } \\
\text { with COVID-19 }(n=13)\end{array}$ \\
\hline \multicolumn{2}{|l|}{ Anesthesia method (No.) (\%) } \\
\hline General anesthesia & $9(69.2 \%)$ \\
\hline Conscious sedation & $4(30.7 \%)$ \\
\hline \multicolumn{2}{|l|}{ Initial treatment approach (No.) (\%) } \\
\hline Stent retriever & $4(30.7 \%)$ \\
\hline ADAPT technique & $6(46.1 \%)$ \\
\hline Combined distal aspiration and stent retriever & $3(23.0 \%)$ \\
\hline \multicolumn{2}{|l|}{ Timing of intervention (median) (IQR)( min) } \\
\hline Femoral puncture to recanalization & $34.5(23-45)$ \\
\hline Symptom onset to recanalization & $298.5(261-324)$ \\
\hline \multicolumn{2}{|l|}{ Angiographic outcome (No.) (\%) } \\
\hline $\mathrm{TICl} 2 \mathrm{~b}-3$ & $10(76.9 \%)$ \\
\hline $\mathrm{TICl} 2 \mathrm{c}-3$ & $9(69.2 \%)$ \\
\hline $\mathrm{TICl} 3$ & $7(53.8 \%)$ \\
\hline \multicolumn{2}{|l|}{ Procedural complications (No.) (\%) } \\
\hline Neurologic & $0(0 \%)$ \\
\hline Non-neurologic (femoral artery occlusion) & $1(7.6 \%)$ \\
\hline \multicolumn{2}{|l|}{ Imaging outcome (No). (\%) } \\
\hline All hemorrhagic transformation & $3(23.0 \%)$ \\
\hline Symptomatic ICH & $0(0 \%)$ \\
\hline $\mathrm{HI}-\mathrm{HI} 2$ & $2(15.3 \%)$ \\
\hline $\mathrm{PH} 1$ & $1(7.6 \%)$ \\
\hline $\mathrm{PH} 2$ & $0(0 \%)$ \\
\hline \multicolumn{2}{|l|}{ Clinical outcome } \\
\hline In-hospital mortality (No.) (\%) & $2(15.3 \%)$ \\
\hline Last available NIHSS (median) (IQR) ${ }^{a}$ & $5(0.5-12.5)$ \\
\hline NIHSS drop (median) (IQR) ${ }^{\mathrm{a}}$ & $2(0.5-5)$ \\
\hline
\end{tabular}

Note:-ADAPT indicates direct aspiration first pass technique; ICH, intracerebral hemorrhage; $\mathrm{PH}$, parenchymal hematoma; HI, petechial hemorrhage.

${ }^{a} n=10$ (excluding 2 deceased patients).
All 3 centers use MR imaging for patient selection. During the COVID19 outbreak, all hospitals were able to maintain around-the-clock care for stroke emergencies.

\section{Statistical Analysis}

Given the small sample size, the analysis was mainly performed using descriptive statistics. Continuous variables are presented as median with interquartile range. Categoric variables are presented as numbers with percentages. Comparisons of time delays were performed using the Mann-Whitney $U$ test after the normality of the distributions was assessed graphically and using the Shapiro-Wilk test. Analyses were performed using GraphPad Prism, Version 6.0 (GraphPad Software).

\section{RESULTS}

We identified 13 patients with SARSCov-2 infection, representing $38.2 \%$ of a total of 34 thrombectomies performed in the 2 centers during the study period. Apart from 1 case, recorded on March 12, all other cases were recorded after March 25.

Baseline characteristics are detailed in Table 1. The median patient age was 78 years, and the median time from symptom onset to groin puncture was 244 minutes. Most cases were anterior circulation strokes $(8 / 13 ; 61.5 \%)$. The median ASPECTS was 8 , and none of the patients had ASPECTS $<5$.

Procedural metrics and outcomes are detailed in Table 2. The median time from groin puncture to recanalization was 34.5 minutes, and TICI $2 \mathrm{~b}-$ 3 recanalization was obtained in 10/13 (76.9\%) cases. No symptomatic hemorrhagic transformation was observed on 24-hour CT scans. In 1 case (7.6\%), 9 hours after successful recanalization of a middle cerebral artery occlusion, we observed early arterial re-occlusion of both internal carotid arteries and of the basilar trunk.

Three patients (23.0\%) were diagnosed with deep vein thrombosis, and 1 (7.6\%), with a pulmonary embolism during hospitalization. Two patients died, accounting for a mortality rate of $15.3 \%$. 
March 1 to April 26, 2019 March 1 to April 26, 2020

Volume of mechanical thrombectomies
Symptom onset to brain imaging (median) (IQR) (min)
Symptom onset to IV thrombolysis ${ }^{\mathrm{a}}$ (median) (IQR) (min)
Imaging to femoral puncture (median) (IQR) (min)
Symptom onset to femoral puncture (median) (IQR) (min)
Femoral puncture to recanalization (median) (IQR) (min)
${ }^{\text {a }}$ For patients who received IV thrombolysis before thrombectomy.

\section{COVID-19 Diagnosis}

Only 1 patient had been diagnosed with COVID-19 before thrombectomy, and the patient was already hospitalized at the time of the stroke. In the remaining 12/13 (92.3\%) cases, patients presented to the hospital primarily for symptoms of acute stroke. On initial examination and history, there were symptoms suggestive of COVID-19 in only 4/12 (33.3\%) cases.

Infection with SARS-Cov-2 was confirmed by reverse transcription polymerase chain reaction in $10 / 13$ patients. In 3 of these cases, the results of the first oropharyngeal swab were negative. Subsequently, 2 patients had a second oropharyngeal swab positive for COVID-19, while for the third patient, the reverse transcription polymerase chain reaction was positive for COVID19 in a sputum sample.

In the remaining 3/13 cases with negative findings with oropharyngeal swabs, the diagnosis was made due to association of typical clinical symptoms and chest CT findings.

\section{Personal Protection Measures}

Both centers used personal protection measures during the study period. In 1 center, measures to prevent contamination of personnel were used systematically for all cases, even in the absence of suggestive clinical symptoms. This center (Strasbourg University Hospital) already used systematic general anesthesia for all thrombectomies, and this practice was continued. Orotracheal intubation was performed in the angiography suite, which is equipped with an ISO 7 air-handling unit (capable of clearing $90 \%$ of $0.5-\mu \mathrm{m}$ particles in less than 6 minutes; Unit model RM09/09, manufacturer ROBATHERM, JettingenScheppach, Germany). All personnel in the angiography suite wore enhanced personal protection equipment, including surgical cap, goggles, full gown/gloves, shoe covers, and a filtering facepiece 3 mask. Extubation was performed in the angiography suite; then, the patient was transferred directly to the stroke unit.

The second center continued their usual practice of thrombectomy with the patient under local anesthesia/conscious sedation. Enhanced personal protective equipment was used only for cases with symptoms suggestive of COVID-19 disease.

During the study period, 1 interventional neuroradiologist developed mild respiratory symptoms suggestive of COVID-19. Subsequent CT of the chest revealed unilateral small ground glass opacities compatible with mild COVID-19 pneumonia. Findings of nasopharyngeal swabs 10 days apart were negative, there was full resolution of symptoms without specific treatment, and the physician resumed activity after 14 days of home confinement. One radiology technician developed respiratory symptoms, tested positive on the first nasopharyngeal swab, and was also able to resume activity after 14 days of home confinement. There were no other documented cases of contamination in physicians or angiography suite staff ( 7 interventional neuroradiologists and 29 radiology technicians).

\section{Comparison with Prepandemic Activity}

Compared with the same time period in 2019, we observed a $21 \%$ decrease in stroke thrombectomy volume. Procedural time metrics compared with the prepandemic period are outlined in Table 3. There was no significant difference in prehospital or in-hospital delays, but this comparison is obviously limited by the small sample size.

\section{DISCUSSION}

During the first 2 months of the COVID-19 outbreak, more than one-third of the patients who underwent stroke thrombectomies had SARS-Cov-2 infection. In most cases, patients did not have a COVID-19 diagnosis or suspicion before the acute presentation with stroke symptoms. Moreover, only 1 of 3 patients had suggestive symptoms at the moment of the acute presentation.

These results underline the importance of personal protection measures for all stroke centers in areas touched by the COVID-19 pandemic. An important proportion of cases were not suspected of having COVID-19 at presentation. This scenario can be explained, on one hand, by the high rate of asymptomatic infections and, on the other hand because the initial examination and history are performed in an expedited fashion in the context of stroke alerts because of time constraints and impaired communication due to neurologic deficits. Consequently, it seems justified to systematically apply full personal protection measures for all stroke thrombectomies, for the entire duration of the epidemic. Recent guidance issued by the Society of NeuroInterventional Surgery ${ }^{3}$ also recommends that patients with undocumented COVID-19 status should be treated as high risk, provided institutional resources are available. However, the high rate of patients positive for COVID-19 observed in our series might not be applicable in other geographic areas. Moreover, the definition of appropriate personal protection measures may vary among facilities and practitioners. This is reflected in the recommendations of the European Society of Minimally Invasive Neurological Therapy, ${ }^{4}$ which state that clinical management should be conducted according to local hospital protocols.

Baseline characteristics among treated cases, as well the duration of procedures and recanalization rates, are comparable with those in previously published literature. ${ }^{5}$ Two recent reports ${ }^{6,7}$ of 
10 thrombectomies in relatively young patients ( 5 cases in each report) suggested that SARS-Cov-2 infection might lead to increased rates of stroke in the younger population. We did not observe this tendency in our cohort. The youngest patient was 62 years of age, and the median age was 78 years, which is above the average age in recent thrombectomy trials. Escalard et $\mathrm{al}^{8}$ recently published their experience of thrombectomy in patients with COVID-19 in a high-volume center in Paris, France. They treated 10 patients positive for COVID-19, representing $27 \%$ of the total 37 thrombectomies performed during the study period; most patients $(70 \%)$ had mild or no respiratory signs at the time of stroke. These findings are comparable with the results in our cohort.

With regard to clinical outcomes, despite the obvious limitations pertaining to the small sample size and short follow-up, out initial results suggest an increase in the in-hospital mortality rate $(15.3 \%)$ and a high rate of thrombotic complications during hospitalization (23.0\% with deep vein thrombosis, $7.6 \%$ with pulmonary embolism, and $7.6 \%$ with early arterial reocclusion). In the cohort of Escalard et al, ${ }^{8}$ these rates were even higher; $40 \%$ of cases had early cerebral re-occlusion and $60 \%$ mortality. This finding is concordant with recent reports indicating a hypercoagulable state in patients with COVID-19. Multiple groups have observed high levels of thrombosis and inflammation serum markers such as D-dimers, fibrinogen, and C-reactive protein. $^{9-11}$ Similarly, a recent study ${ }^{12}$ found that pulmonary embolism is frequent and is seen in almost 1 of 4 patients with severe clinical features of COVID-19 infection.

Given this physiopathologic evidence, we should be seeing an increase in the incidence of ischemic stroke. Initial reports seem to point in the opposite direction: Two centers in northern Italy ${ }^{13,14}$ observed a reduction in the number of acute presentations, stroke admissions, and the proportion of patients treated with intravenous thrombolysis or mechanical thrombectomy. In our region, compared with the same period in 2019, between March 1 and 31, 2020, there were 39.6\% fewer stroke alerts and $33.3 \%$ fewer acute revascularization treatments (intravenous thrombolysis and/or mechanical thrombectomy), with a relatively stable number of stroke unit admissions $(-0.6 \%) .{ }^{15}$ This finding suggests that the overall stroke incidence remained the same, but fewer patients presented within the therapeutic time window and were thus eligible for intravenous thrombolysis or mechanical thrombectomy.

The incidence of ischemic stroke among patients with COVID-19 remains unknown. In our center, ${ }^{16}$ brain MR imaging was performed in 13 patients hospitalized in the intensive care unit with severe COVID-19 infection and acute respiratory distress syndrome. Three cases (23\%) had evidence of acute or subacute ischemic stroke. Of note, these patients did not have focal signs suggestive of stroke; they underwent MR imaging because of unexplained encephalopathic features. These findings suggest that ischemic stroke might be frequent in patients with severe COVID-19 infection, but a large proportion of cases are not detected because clinical symptoms are masked by the severity of respiratory features and sedation.
The main limitations of this study are the small sample size and the absence of a comparative cohort. However, the aim of this study was primarily to describe our initial experience of stroke thrombectomy in this particular context and discuss possible implications for clinical practice.

\section{CONCLUSIONS}

More than one-third of patients who underwent stroke thrombectomies during the epidemic had SARS-Cov-2 infection. Most patients had not been diagnosed or suspected before the acute stroke presentation, and often they did not have suggestive symptoms on initial examination. In view of these results, it seems justified to systematically apply full personal protection measures for all stroke thrombectomies performed during this epidemic. Patient characteristics and thrombectomy metrics were comparable with those in recent clinical trials; however, initial data on clinical outcomes suggest increased mortality and a high incidence of thrombotic complications during hospitalization.

Disclosures: Julien Pottecher—UNRELATED: Consultancy: LFB BIOMEDICAMENTS; Payment for Development of Educational Presentations: Baxter. Thiên-Nga Chamaraux-Tran—UNRELATED: Grants/Grants Pending: Société Française d'Anesthésie de et Réanimation, Comments: research grant; Payment for Lectures Including Service on Speakers Bureaus: Merck Sharp \& Dohme, LFB. Francois SellalUNRELATED: Payment for Lectures Including Service on Speakers Bureaus: Novartis Pharmaceuticals, Sanofi, Crédit Mutuel.

\section{REFERENCES}

1. Bernard Stoecklin S, Rolland P, Silue Y, et al; Investigation Team. First cases of coronavirus disease 2019 (COVID-19) in France: surveillance, investigations and control measures, January 2020. Euro Surveill 2020;25:200009 CrossRef Medline

2. COVID-19-France. https://www.gouvernement.fr/info-coronavirus/ carte-et-donnees. Accessed April 17, 2020

3. Fraser JF, Arthur AS, Chen M, et al. Society of NeuroInterventional Surgery recommendations for the care of emergent neurointerventional patients in the setting of COVID-19. J Neurointerv Surg 2020;12:539-41 CrossRef Medline

4. Aggour M, White P, Kulcsar Z, et al. European Society of Minimally Invasive Neurological Therapy (ESMINT) recommendations for optimal interventional neurovascular management in the COVID-19 era. J Neurointerv Surg 2020;12:542-44 CrossRef Medline

5. Goyal M, Menon BK, van Zwam WH, et al; HERMES collaborators. Endovascular thrombectomy after large-vessel ischaemic stroke: a meta-analysis of individual patient data from five randomised trials. Lancet 2016;387:1723-31 CrossRef Medline

6. Oxley TJ, Mocco J, Majidi S, et al. Large-vessel stroke as a presenting feature of Covid-19 in the young. N Engl J Med 2020;382:e60 CrossRef Medline

7. Wang A, Mandigo GK, Yim PD, et al. Stroke and mechanical thrombectomy in patients with COVID-19: technical observations and patient characteristics. J Neurointerv Surg 2020;12:648-53 CrossRef Medline

8. Escalard S, Maïer B, Redjem H, et al. Treatment of acute ischemic stroke due to large vessel occlusion with COVID-19: experience from Paris. Stroke 2020;51:2540-43 CrossRef Medline

9. Li Y, Wang M, Zhou Y, et al. Acute cerebrovascular disease following COVID-19: a single center, retrospective, observational study. Stroke Vasc Neurol 2020 Jul 2. [Epub ahead of print] CrossRef Medline

10. Madjid M, Safavi-Naeini P, Solomon SD, et al. Potential effects of coronaviruses on the cardiovascular system: a review. JAMA Cardiology 2020 March 27. [Epub ahead of print] CrossRef Medline 
11. Mehta P, McAuley DF, Brown M, et al; HLH Across Speciality Collaboration, UK. COVID-19: consider cytokine storm syndromes and immunosuppression. Lancet 2020;395:1033-34 CrossRef Medline

12. Grillet F, Behr J, Calame P, et al. Acute pulmonary embolism associated with covid-19 pneumonia detected by pulmonary CT angiography. Radiology 2020 April 23. [Epub ahead of print] CrossRef Medline

13. Baracchini C, Pieroni A, Viaro F, et al. Acute stroke management pathway during Coronavirus-19 pandemic. Neurol Sci 2020;41:100305 CrossRef Medline
14. Morelli N, Rota E, Terracciano C, et al. The baffling case of ischemic stroke disappearance from the casualty department in the COVID19 era. Eur Neurol 2020;83:213-15 CrossRef Medline

15. Pop R, Quenardelle V, Hasiu A, et al. Impact of the Covid-19 outbreak on acute stroke pathways - Insights from the Alsace region in France. Eur J Neurol 2020 May 12. [Epub ahead of print] CrossRef Medline

16. Helms J, Kremer S, Merdji H, et al. Neurologic features in severe SARS-CoV-2 infection. $N$ Engl J Med 2020;382:2268-70 CrossRef Medline 